\title{
Metastatic Malignant Neoplasm in the Abdominal Cavity
}

National Cancer Institute

\section{Source}

National Cancer Institute. Metastatic Malignant Neoplasm in the Abdominal Cavity. NCI Thesaurus. Code C8615.

The spread of a malignant neoplasm from a primary site to an unspecified organ in the abdominal cavity. 